PROCEEDINGS OF THE

AMERICAN MATHEMATICAL SOCIETY

Volume 127, Number 5, Pages 1493-1497

S 0002-9939(99)04695-X

Article electronically published on February 4, 1999

\title{
THE LOCATION OF THE ZEROS OF THE HIGHER ORDER DERIVATIVES OF A POLYNOMIAL
}

\author{
PIOTR PAWLOWSKI
}

(Communicated by Theodore W. Gamelin)

\begin{abstract}
Let $p(z)$ be a complex polynomial of degree $n$ having $k$ zeros in a disk $D$. We deal with the problem of finding the smallest concentric disk containing $k-l$ zeros of $p^{(l)}(z)$. We obtain some estimates on the radius of this disk in general as well as in the special case, where $k$ zeros in $D$ are isolated from the other zeros of $p(z)$. We indicate an application to the root-finding algorithms.
\end{abstract}

\section{INTRODUCTION}

Let us consider the following problem: If $k$ zeros of a polynomial $p(z)$ of degree $n(2 \leq k \leq n)$ lie in a disk $D$ of radius $r$, what is the smallest concentric disk that contains $k-l$ zeros of $p^{(l)}(z)(1 \leq l \leq k-1)$ ? Since the problem is scaling and translation invariant, we can assume that the disk $D$ is the closed unit disk $\bar{\Delta}:=\{z \in \mathbb{C}:|z| \leq 1\}$. Let $\mathcal{P}_{n, k}$ denote the class of complex polynomials of degree $n$ having exactly $k$ zeros in $\bar{\Delta}$. We define the function $\rho(n, k, l), n \geq k>l$, as follows:

$$
\rho(n, k, l)=\sup _{p \in \mathcal{P}_{n, k}} \min \left\{R>0: \bar{D}(0, R) \text { contains at least } k-l \text { zeros of } p^{(l)}(z)\right\} .
$$

Because of scaling and translation invariance we can conclude that if $\bar{D}(c, r)$ contains $k$ zeros of the polynomial $p(z)$, then $\bar{D}(c, r \rho(n, k, l))$ contains $k-l$ zeros of $p^{(l)}(z)$.

The problem of estimating $\rho(n, k, l)$ has a long history in the case $l=1$. The results listed below can be found in Marden's book ([2]). The Gauss-Lucas Theorem states that $\rho(n, n, 1)=1$. Result $\rho(n, 2,1)=\cot (\pi / n)$ is due to Alexander, Kakeya and Szegö. Biernacki proved that

$$
\rho(n, n-1,1) \leq(1+1 / n)^{1 / 2} \quad \text { and } \quad \rho(n, k, 1) \leq \prod_{i=1}^{n-k}[(n+i) /(n-i),
$$

and Marden showed that

$$
\rho(n, k, 1) \leq \csc \frac{\pi}{2(n-k+1)} .
$$

Received by the editors February 5, 1997 and, in revised form, September 3, 1997. 1991 Mathematics Subject Classification. Primary 30C15; Secondary 65E05.

(C)1999 American Mathematical Society 
More recently, Coppersmith and Neff ([1]) proved that, under the condition that $k$ zeros in $\bar{\Delta}$ are centered at 0 ,

$$
\rho(n, k, l) \leq 10.477 \frac{(n-k+1)(k-l)}{\sqrt{k}}
$$

and

$$
\rho(n, k, k-1) \leq C \max \left\{(n-k+1)^{1 / 2} k^{-1 / 4},(n-k+1) k^{-2 / 3}\right\} .
$$

One can also impose an additional condition on $p \in \mathcal{P}_{n, k}$ to have zeros in $\Delta$ isolated from the other zeros. Following Pan ([3]), we define the isolation ratio of a polynomial $p$ with respect to a disk $D, I(p, D)$, as:

$$
\begin{aligned}
I(p, D(c, r)):=\sup \{\mu>0: D(c, \mu r) \text { contains exactly } \\
\text { the same zeros of } p \text { as } D(c, r)\} .
\end{aligned}
$$

We say that a disk $D$ is $f$-isolated if $I(p, D) \geq f$. The isolation ratio is also scaling and translation invariant. We define the isolation ratio of $p$ as $I(p):=I(p, \bar{\Delta})$. Renegar ([5]) proved the following result:

$$
\text { If } I(p) \geq 15 n^{3} \text {, then } \rho(n, k, k-1) \leq \frac{3 n}{2} \text { and } I\left(p^{(k-1)}, \bar{D}(0,3 n / 2)\right) \geq \frac{I(p)}{10 n^{2}} \text {. }
$$

In other words, if $k$ zeros of a polynomial $p$ are well-isolated in some disk, then $p^{(k-1)}$ has an isolated single zero in a larger concentric disk. Using Walsh's Coincidence Theorem ([2]) we improve the bound in (1.7). We also generalize Biernacki's proof of (1.2) and obtain an upper bound for $\rho(n, k, l)$ which is smaller than (1.4) for some special choices of $n, k$ and $l$.

Results of this type have found applications in constructing low complexity algorithms for finding zeros of polynomials. Smale and Renegar ([7], [5]) derived quantitative criteria for a point to be in the domain of quadratic convergence of Newton's algorithm. The basic idea is that if a zero is isolated from other zeros, then we need only crude approximation to obtain fast convergence. However, if we have an isolated cluster of $k$ zeros, then $p^{(k-1)}$ should have a single zero nearby. In this case, the result like (1.7) allows us to apply Newton's algorithm to $p^{(k-1)}$ with quadratic convergence. To establish initial isolation of clusters of zeros, various global search algorithms are used ([4], [5])

\section{Main Results}

First we prove the following proposition:

\section{Proposition 2.1.}

$$
\begin{aligned}
& \text { If } I(p) \geq 1+2 \frac{l(n-k)}{k-l+1} \text {, then } \rho(n, k, l) \leq 1 \text { and } \\
& \qquad I\left(p^{(l)}\right) \geq \frac{(k-l+1) I(p)-l(n-k)}{k-l+1+l(n-k)} .
\end{aligned}
$$

Proof. Let $I(p)=R$ and let $p(z)=f(z) g(z)$ where $f(z)$ is a polynomial of degree $k$ whose zeros lie in $\bar{\Delta}$ and $g(z)$ is a polynomial of degree $n-k$ whose zeros lie in 
$\{z:|z|>R\}$. Let $m=\min (n-k, l)$. If $z$ is a solution of the equation

$$
p^{(l)}(z)=\sum_{i=0}^{m}\left(\begin{array}{l}
l \\
i
\end{array}\right) g^{(i)}(z) f^{(l-i)}(z)=0,
$$

then the Walsh Coincidence Theorem implies that there exist $x_{1},\left|x_{1}\right| \leq 1$ and $x_{2},\left|x_{2}\right| \geq R$ such that

$$
\sum_{i=0}^{m}\left(\begin{array}{l}
l \\
i
\end{array}\right)\left(\left(z-x_{2}\right)^{n-k}\right)^{(i)}\left(\left(z-x_{1}\right)^{k}\right)^{(l-i)}=0
$$

i.e.

$$
\sum_{i=0}^{m}\left(\begin{array}{l}
l \\
i
\end{array}\right) \frac{(n-k) !}{(n-k-i) !}\left(z-x_{2}\right)^{n-k-i} \frac{k !}{(k-l+i) !}\left(z-x_{1}\right)^{k-l+i}=0
$$

i.e.

$$
\left(z-x_{1}\right)^{k-l}\left(z-x_{2}\right)^{n-k-m} \sum_{i=0}^{m}\left(\begin{array}{l}
l \\
i
\end{array}\right)\left(\begin{array}{c}
n-l \\
n-k-i
\end{array}\right)\left(z-x_{2}\right)^{m-i}\left(z-x_{1}\right)^{i}=0 .
$$

Also $z$ must lie in the interval $\left[x_{1}, x_{2}\right]$ and either $z=x_{1}$ or $z=x_{2}$ or $z$ is a zero of the polynomial

$$
q(z)=\sum_{i=0}^{m}\left(\begin{array}{l}
l \\
i
\end{array}\right)\left(\begin{array}{c}
n-l \\
n-k-i
\end{array}\right)\left(z-x_{2}\right)^{m-i}\left(z-x_{1}\right)^{i} .
$$

Let $w=\frac{z-x_{1}}{z-x_{2}}$ and let

$$
h(w)=\sum_{i=0}^{m}\left(\begin{array}{l}
l \\
i
\end{array}\right)\left(\begin{array}{c}
n-l \\
n-k-i
\end{array}\right) w^{i}=\sum_{i=0}^{m} a_{i} w^{i} .
$$

All zeros of $h(w)$ lie on the negative real axis and by the Enestrőm-Kakeya Theorem they satisfy the inequality

$$
\min _{0 \leq i \leq m-1} \frac{a_{i}}{a_{i+1}} \leq|w| \leq \max _{0 \leq i \leq m-1} \frac{a_{i}}{a_{i+1}} .
$$

We have that

$$
\frac{a_{i}}{a_{i+1}}=\frac{(i+1)(k-l+i+1)}{(l-i)(n-k-i)}
$$

therefore

$$
\zeta:=\min _{0 \leq i \leq m-1} \frac{a_{i}}{a_{i+1}}=\frac{k-l+1}{l(n-k)}
$$

and

$$
\xi:=\max _{0 \leq i \leq m-1} \frac{a_{i}}{a_{i+1}}=\frac{m(m+k-l)}{(l-m+1)(n-k-m+1)} .
$$

Since all zeros of $h(w)$ lie in the interval $[-\xi,-\zeta]$, all zeros of $q(z)$ lie in the interval $\left[\frac{\zeta x_{2}+x_{1}}{\zeta+1}, \frac{\xi x_{2}+x_{1}}{\xi+1}\right]$. By continuity, $p^{(l)}(z)$ has $k-l$ zeros in $\bar{\Delta}$ if $\left|\frac{\zeta x_{2}+x_{1}}{\zeta+1}\right| \geq 1$. Since 
$\left|\frac{\zeta x_{2}+x_{1}}{\zeta+1}\right| \leq \frac{\zeta R-1}{\zeta+1}$, the above inequality holds if $\frac{\zeta R-1}{\zeta+1} \geq 1$, i.e. if $R \geq\left(1+\frac{2}{\zeta}\right)=$ $\left(1+2 \frac{l(n-k)}{k-l+1}\right)$. These are the only zeros of $p^{(l)}(z)$ in the disk

$$
D\left(0, \frac{\zeta R-1}{\zeta+1}\right)=D\left(0, \frac{(k-l+1) R-l(n-k)}{k-l+1+l(n-k)}\right) .
$$

We use Proposition 2.1 to prove the following result analogous to (1.7).

\section{Theorem 2.2.}

$$
\text { If } I(p) \geq \frac{n^{2}}{4}, \text { then } \rho(n, k, k-1) \leq 1 \text { and } I\left(p^{(k-1)}\right) \geq \frac{4 I(p)}{n^{2}} .
$$

Proof. If $l=k-1$ and $I(p) \geq 1+(k-1)(n-k)$, then, by Proposition $2.1, \bar{\Delta}$ contains a zero of $p^{(k-1)}$. Since $1+(k-1)(n-k) \leq k(n-k) \leq n^{2} / 4$, the same is true if $I(p) \geq n^{2} / 4$. Also,

$$
I\left(p^{(k-1)}\right) \geq \frac{2 I(p)-(k-1)(n-k)}{2+(k-1)(n-k)}>\frac{I(p)}{1+(k-1)(n-k)} \geq \frac{I(p)}{k(n-k)} \geq \frac{4 I(p)}{n^{2}} .
$$

Remark 2.3. We don't know of any estimates for $\rho(n, k, k-1)$ if the isolation ratio of $p$ is smaller than $n^{2} / 4$. In applications to zero-finding we can use the classical Graeffe process of root-squaring to control the isolation ratio of a polynomial ([3],[6]). If $p_{0}$ has zeros $z_{1}, \ldots, z_{n}$ and isolation ratio $C>1$, then the polynomial

$$
p_{1}(z):=p_{0}(\sqrt{z}) p_{0}(-\sqrt{z})
$$

has zeros $z_{1}^{2}, \ldots, z_{n}^{2}$ and isolation ratio $C^{2}$. By iterating this process we obtain polynomial $p_{m}$ with zeros $z_{1}^{2^{m}}, \ldots, z_{n}^{2 m}$ and isolation ratio $C^{2^{m}}$.

We can also apply Proposition 2.1 to obtain an upper bound for $\rho(n, k, l)$.

\section{Theorem 2.4.}

$$
\rho(n, k, l) \leq \frac{n+l}{n-l} \prod_{i=k}^{n-2}\left(1+\frac{2 l(n-i)}{i-l+1}\right)
$$

Proof. Let us assume that the zeros of $p(z)$ are ordered by their moduli, $\left|z_{1}\right| \leq\left|z_{2}\right| \leq$ $\ldots \leq\left|z_{n}\right|$. If $k=n-1$, then $p(z)=f(z)\left(z-z_{n}\right)$, where $f(z)$ is a polynomial with zeros $z_{i},\left|z_{i}\right| \leq 1,1 \leq i \leq n-1$. We have

$$
p^{(l)}(z)=f^{(l)}(z)\left(z-z_{n}\right)+l f^{(l-1)}(z) .
$$

By the Walsh Coincidence Theorem, if $z$ is a zero of $p^{(l)}(z)$ it is also a zero of the equation:

$$
\left((z-\gamma)^{n-1}\right)^{(l)}(z)\left(z-z_{n}\right)+l\left((z-\gamma)^{n-1}\right)^{(l-1)}(z)=0
$$

where $|\gamma| \leq 1$, i.e.,

$$
(z-\gamma)^{n-1-l}\left((n-l)\left(z-z_{n}\right)+l(z-\gamma)\right)=0 .
$$


Therefore $z=\gamma$ or $z=\left((n-l) z_{n}+l \gamma\right) / n$. Also if $\left|\left((n-l) z_{n}+l \gamma\right) / n\right|>1$, then there are exactly $k-1-l$ zeros of $p^{(l)}(z)$ in $\bar{\Delta}$. This is the case if $\left|z_{n}\right|>\frac{n+l}{n-l}$. Otherwise, by the Gauss-Lucas Theorem, all zeros of $p^{(l)}(z)$ are in the disk $D\left(0, \frac{n+l}{n-l}\right)$. Now let's fix $K$ and suppose that (2.16) holds for $k=n-1 \ldots K-1$. By Proposition 2.1 , if $\left|z_{K+1}\right|>1+2 \frac{l(n-K)}{K-l+1}$, then there are exactly $K-l$ zeros of $p^{(l)}(z)$ in $\bar{\Delta}$. Otherwise there are $K+1$ zeros of $p(z)$ in the disk $D\left(0,1+2 \frac{l(n-K)}{K-l+1}\right)$ and by induction there are at least $K-l$ zeros of $p^{(l)}(z)$ in $D(0, R)$, where

$$
R \leq \frac{n+l}{n-l} \prod_{i=K}^{n-2}\left(1+\frac{2 l(n-i)}{i-l+1}\right) .
$$

Inequality (2.16) can be better in some cases than (1.4), namely if $k$ and $l$ are close to $n$. In the most interesting case $l=k-1$, Coppersmith and Neff gave a lower bound on $\rho(n, k, k-1)$ and also conjectured that if $k>n / 2$, then $\rho(n, k, k-1)$ is bounded by a constant.

\section{REFERENCES}

[1] D. Coppersmith, C.A. Neff, Roots of a Polynomial and its Derivatives. Proceedings of the Fifth Annual ACM-SIAM Symposium on Discrete Algorithms (Arlington, VA, 1994), 271279, ACM, New York, 1994. MR 95c:30008

[2] M. Marden, Geometry of Polynomials. Math. Surveys 3, Amer. Math. Soc. Providence, R.I. 1966. MR 37:1562

[3] V.Y. Pan, New Techniques for Approximating Complex Polynomial Zeros Proceedings of the Fifth Annual ACM-SIAM Symposium on Discrete Algorithms (Arlington, VA, 1994), 260-270, ACM, New York, 1994. MR 95g:65194

[4] V.Y. Pan, Sequential and Parallel Complexity of Approximate Evaluation of Polynomial Zeros Computers and Math. (with Applications), 14 (1987) 8, 591-622. MR 88j:65101

[5] J. Renegar, On the worst-case arithmetic complexity of approximating zeros of polynomials. Journal of Complexity, 3 (1987) 90-113. MR 89a:68107

[6] A. Schőnhage, Equation Solving in Terms of Computational Complexity, Proceedings of the International Congress of Mathematicians, Berkeley, California, 1986. MR 89h:68071

[7] S. Smale, Newton's method estimates from data at one point, The Merging Disciplines: New Directions in Pure, Applied and Computational Mathematics. 185-196, Springer-Verlag, 1986. MR 88e: 65076

Department of Mathematics and Computer Science, Kent State University, Kent, OHIO 44242

E-mail address: ppawlows@mcs.kent.edu

Current address: Summit Systems, Inc., 22 Cortlandt Street, New York, New York 10007

E-mail address: piotr_pawlowski@summithq.com 